\title{
Crystal structure of $\mathrm{Ce}_{0.8} \mathrm{Ho}_{0.1} \mathrm{Gd}_{0.1} \mathrm{O}_{1.9}$ between $295 \mathrm{~K}$ and $1023 \mathrm{~K}$
}

\section{Sri Rahayu, Jennifer S. Forrester, Girish M. Kale* and Mojtaba Ghadiri}

School of Chemical and Process Engineering, University of Leeds, Leeds LS2 9JT, United Kingdom

Correspondence email: G.M.Kale@leeds.ac.uk

\begin{abstract}
The crystal structure of $\mathrm{Ce}_{0.8} \mathrm{Ho}_{0.1} \mathrm{Gd}_{0.1} \mathrm{O}_{1.9}$ (Cerium Holmium Gadolinium Oxide) is determined from powder X-ray diffraction data. This is a promising material for potential application as a solid electrolyte for intermediate temperature solid oxide fuel cells (IT-SOFC). Nanoparticles were prepared using a novel sodium alginate sol gel method, where the sodium ion was exchanged with ions of interest, and after washing the gel was calcined at $723 \mathrm{~K}$ in air. The crystallographic features of Ho and Gd co-doped Cerium Oxide were investigated around the desired operating temperatures of intermediate-temperature solid-oxide fuel cells, $573 \mathrm{~K} \leq \mathrm{T} \leq 1023 \mathrm{~K}$. We find that the crystal structure is a stable fluorite structure with space group F $\mathrm{m}-3 \mathrm{~m}$ in the entire temperature range. In addition, the trend in lattice parameters shows that there is a monotonic increase with increasing temperature. The aim of this study is to investigate the crystal structure around the operation temperature of IT-SOFC co-doped Cerium Gadolinium Oxide using in situ X-ray diffraction.
\end{abstract}

\section{Introduction}

The stability of the crystallographic structure of Cerium Oxide co-doped with gadolinium and holmium is important at operating temperatures of 723-923 K (Artini et al., 2014). Cerium Holmium Gadolinium Oxide is a promising material for use as the solid electrolyte in solid oxide fuel cells because two dissimilar trivalent substitutional cations (co-doping) can be used to minimize the activation energy and optimize the conductivity at intermediate temperatures (723-923 K) (Kilner \& Brook, 1982; Kilner \& Waters, 1982; Steele, 2000; Kilner \& Burriel, 2014; Rushton et al., 2013). Measuring changes in the lattice parameters as a function of the dopant concentration is one way to evaluate the minimization of oxygen vacancy trapping (Kilner, 1983). Lattice simulations have been developed that have led to the recognition of the importance of elastic energy terms due to size mismatch of the dopant cations (Kilner, 1983). Kim (1989) proposed an empirical equation between dopant size and ionic conductivity; and the concept of critical ion radius of a dopant was developed. The critical ionic radius for a trivalent dopant in Ceria is $0.1038 \mathrm{~nm}$, which is close to the ionic radius of $\mathrm{Gd}^{3+}([\mathrm{C} \cdot \mathrm{N} .: 6] 0.9382 \AA$ ) (Artini et al., 2012; Kim, 1989; Shannon \& Prewitt, 1968). Kilner suggested that the conductivity is affected by ionic size mismatch between the host and the dopant, in that a smaller ionic size mismatch allows a higher conductivity to be achieved (Kilner \& Brook, 1982; Kilner \& Waters 1982).

Ou et al. (2007) reported that microstructural inhomogeneity has a negative effect on the ionic conductivity of a material due to the interactions between the segregated dopant cations and the oxygen vacancies. Phase homogeneity is a major priority because the oxygen conduction path is mostly aligned along the oxygen vacancies. An inhomogeneous oxygen conduction path can produce isolated oxygen vacancies, which do not contribute to the oxygen conductivity. Non-Vegard behavior in lattice parameters has been observed in the compounds $\mathrm{Ce}-\mathrm{Dy}, \mathrm{Ce}-\mathrm{Ho}$ and $\mathrm{Ce}-\mathrm{Gd}$ mixed oxide structures (Artini et al., 2014; Chavan \& Tyagi, 2005).

The parent structure of $\mathrm{CeO}_{2}$ is a fluorite cubic structure with space group $\mathrm{F} \mathrm{m}-3 \mathrm{~m}$. This structure contains four $\mathrm{CeO}_{2}$ formula units per cell and has lattice parameter $\mathrm{a}=5.4113 \AA$. The structure has a highly symmetric atomic arrangement, i.e., $\mathrm{Ce}$ at $(0,0,0)$ and $\mathrm{O}$ at $\left(1 / 4 \frac{1}{4} \frac{1}{4}\right)$. In addition, $\mathrm{Gd}_{2} \mathrm{O}_{3}$ crystallizes in the cubic structure containing one-fourth $\mathrm{O}$ atoms less than $\mathrm{CeO}_{2}$. This structure belongs to the $\mathrm{I}$ a -3 space group and has a lattice parameter twice as large as $\mathrm{CeO}_{2}$ (10.823 $\AA$ ). $\mathrm{Ho}_{2} \mathrm{O}_{3}$ has a similar crystal structure to $\mathrm{Gd}_{2} \mathrm{O}_{3}$. The lattice parameter of $\mathrm{Ho}_{2} \mathrm{O}_{3}$ is smaller than $\mathrm{Gd}_{2} \mathrm{O}_{3}(10.6050$ 
Å) (Artini et al., 2012; Artini et al., 2014; Grover \& Tyagi, 2004; Maslen et al., 1996; Nakagawa et al., 2001). The structural features of Cerium Gadolinium Oxide are well known; however a study of co-doped Cerium Oxide is useful for understanding the effect of co-doping $\mathrm{Ho}^{3+}$ and $\mathrm{Gd}^{3+}$ on the cation sublattice, and the crystallographic features especially around the operating temperatures of IT-SOFC. This study has not been previously undertaken and therefore provides a valuable input to the area of novel crystal structures in solid oxide fuel cells.

\section{Experimental}

Nanoparticles of $\mathrm{Ce}_{0.8} \mathrm{Ho}_{0.1} \mathrm{Gd}_{0.1} \mathrm{O}_{1.9}$ were synthesized using sodium alginate (SAL) beads, by ion exchange of the sodium ion with these ion of interest (Wang et al., 2012; Wang et al., 2012). The SAL solution was prepared by dissolving stoichiometric amounts of SAL powder in distilled water using a magnetic stirrer with a 500 r.p.m. stirring rate. The metal complex solution was produced by dissolving stoichiometric amounts of respective metal nitrates into $500 \mathrm{ml}$ distilled water which was stirred (500 r.p.m.) with the magnetic stirrer. The SAL solution was dripped into the metal complex solution, which instantly transformed into gel beads. The alginate beads were maintained in the gelling phase for $14 \mathrm{~h}$ under slow magnetic stirring in the nitrate solution to facilitate the ion exchange reaction between $\mathrm{Na}^{+}$with metal ions in the complex salt solution. Following the ion exchange process, the wet metal-alginate beads were separated from the solution using a stainless steel sieve, followed by washing with distilled water and drying for $24 \mathrm{~h}(\mathrm{~T}=363 \mathrm{~K})$. Dry beads were calcined at $723 \mathrm{~K}$ for $2 \mathrm{~h}$ in air, and during this process the alginate decomposed and was eliminated from the final product. Only oxide powders remained at the end of the process.

The nanoparticles were analyzed by X-ray diffraction (XRD) using a PANalytical $X^{\prime}$ Pert XRD equipped with an Anton Paar HTK heating attachment. The XRD patterns were recorded from $10-100^{\circ} 2 \theta$, in the temperature range $573 \mathrm{~K} \leq \mathrm{T} \leq$ $1023 \mathrm{~K}$. The samples were heated in air using a heating rate of $278 \mathrm{~K} / \mathrm{min}$ and held at each temperature for $30 \mathrm{~min}$ to record the diffraction data.

\section{Results and discussion}

Rietveld analysis was carried out using the program GSAS (Larson \& Von Dreele, 2004). The crystal structure features such as changes in lattice parameters, atomic occupancies, isotropic displacement parameters, scale and four polynomial background parameters were refined. The Rietveld refinement of $\mathrm{Ce}_{0.8} \mathrm{Ho}_{0.1} \mathrm{Gd}_{0.1} \mathrm{O}_{1.9}$ at a typical intermediate temperature $873 \mathrm{~K}$ is shown in Fig. 1.

Figure 2 shows the XRD patterns of Cerium Holmium Gadolinium Oxide at selected temperatures. The structure from room temperature until $1023 \mathrm{~K}$ shows a stable fluoritic structure; there is no second phase or hybrid phase detected in the operation temperature range of IT-SOFC, at least to the resolution of the XRD patterns. The co-doped ions $\mathrm{Gd}^{3+}{\text { and } \mathrm{Ho}^{3+}}^{3+}$ substitute for $\mathrm{Ce}^{4+}$ in the fluoritic structure of $\mathrm{CeO}_{2}$ forming a substitutional solid solution, and as a consequence the position of atoms do not change. The difference between the ionic radii of $\mathrm{Ce}^{4+}$ and $\mathrm{Gd}^{3+} / \mathrm{Ho}^{3+}$ is less than $15 \%$ and therefore the structure obeys Hume-Rothery laws for substitutional solid solutions. This result is similar to the analysis performed by Artini, where it is stated that the substitution of $\mathrm{Gd}^{3+}$ of less than $x=0.2$ in $\mathrm{Ce}_{1-x} M_{x} \mathrm{O}_{1.9}$ adopts the fluorite structure.

The temperature dependence of the lattice parameter shows a polynomial trend, as exhibited in Fig. 3 . The trend in the lattice parameter shows an increase with increasing temperature, which can be modelled by the quadratic polynomial function represented by equation (1). At higher temperatures, the thermal energy results in unit cell expansion as expected for stable oxide materials.

The lattice thermal expansion (\% LTE) of $\mathrm{Ce}_{0.8} \mathrm{Ho}_{0.1} \mathrm{Gd}_{0.1} \mathrm{O}_{1.9}$ was studied from 10-100 2 $\theta$, in the temperature range 573 $\mathrm{K} \leq \mathrm{T} \leq 1023 \mathrm{~K}$. In situ XRD patterns were recorded at different temperatures, as shown in Fig. 2. The maximum change of the lattice parameter as a function of temperature can be calculated using percentage lattice thermal expansion (\% 
LTE):

where $\alpha_{298 \mathrm{~K}}$ is the value of the cell parameter at room temperature and $\alpha_{\mathrm{T}}$ is the value of the cell parameter at a selected temperature in $\AA$ (Artini et al., 2014). In Fig. 4, the \% LTE, calculated using the lattice parameters obtained from the refinements, is shown. The calculated \% lattice thermal expansion (\% LTE) was fitted as a function of temperature using a quadratic polynomial regression analysis as follows:

Chavan and Tyagi (2005) observed thermal expansion in the $\mathrm{Ce}_{0.5} \mathrm{RE}_{0.5} \mathrm{O}_{1.75}(\mathrm{RE}=$ trivalent rare earth ion) system. It was found that the coefficient of thermal expansion (CTE) from the partial substitution of Ce by trivalent ions in the $\mathrm{CeO}_{2} \mathrm{ma}_{-}$ trix was less than pure $\mathrm{CeO}_{2}$. This reduction takes place when a distortion of the structure occurs due to the introduction of oxygen vacancies (defects) in relation to a room temperature value (Chavan \& Tyagi, 2005; Artini, et al., 2014). The CTE is calculated at the highest temperature (1023 K) using equation (4)

The CTE of Cerium Holmium Gadolinium Oxide calculated from the present data using equation (4) is $12.898 \times 10^{-6}$ $\mathrm{K}^{-1}$. This value is close to the CTE of Cerium Oxide $\left(12.68 \times 10^{-6} \mathrm{~K}^{-1}\right)($ Chavan \& Tyagi, 2005). The CTE of Cerium Holmium Gadolinium Oxide is similar to the measured value of pure $\mathrm{CeO} 2$ because the partial substitution of a trivalent ion for Ce to form a solid solution does not cause a substantial distortion in the fluoritic structure (Artini, et al., 2014).

The crystallographic features of Ho and Gd co-doped Cerium based have been investigated around the desired operating temperatures in IT-SOFC. The partial substitution of $\mathrm{Ce}^{4+}$ by a trivalent ion results in a solid solution; the crystal structure shows a stable fluorite structure in the entire measured temperature range. In addition, the trend in lattice parameters shows an increase with increasing temperature. The Coefficient of Thermal Expansion (CTE) does not change substantially because the fluorite-type structure does not develop any remarkable distortion as a result of forming a substitutional solid solution. A negligible change in CTE of the solid electrolyte is important for the stable operation and longer life of an Intermediate Temperature Solid Oxide Fuel Cells (IT-SOFC) by minimizing the compressive stress in sinter solid electrolyte at elevated temperature.

\section{Table 1}

Experimental details

\section{Crystal data}

Chemical formula

$M_{\mathrm{r}}$

Crystal system, space group

Temperature (K)

$a(\AA)$

$V\left(\AA^{3}\right)$

$Z$

Radiation type

Specimen shape, size (mm)

Data collection

Diffractometer

Specimen mounting

Data collection mode

Scan method

$2 \theta$ values $\left(^{\circ}\right)$

Refinement

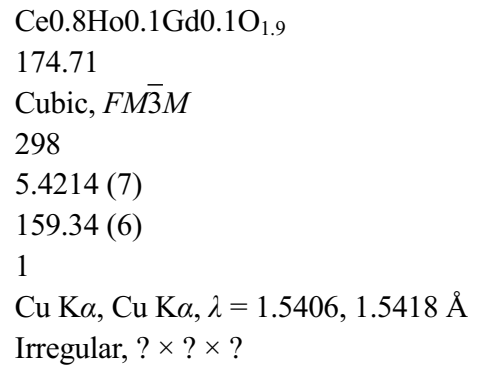


$R$ factors and goodness of fit No. of parameters
$R_{\mathrm{p}}=0.041, R_{\mathrm{wp}}=0.052, R_{\exp }=0.044, R\left(F^{2}\right)=0.04859, \chi^{2}=1.416$

24

Computer programs: GSAS, Atoms 6.2, enCIFer.

\section{Acknowledgements}

$\mathrm{S} R$ would like to thank the Ministry of Research, Technology, and Higher Education of Republic of Indonesia for the scholarship through Riset-Pro.

\section{Funding information}

\section{References}

Aguadero, A., Fawcett, L., Taub, S., Woolley, R., Wu, K.-T., Xu, N., Kilner, J. A. \& Skinner, S. J. (2012). J. Mater. Sci.

47, 3925-3948.

Artini, C., Costa, G. A., Pani, M., Lausi, A. \& Plaisier, J. (2012). J. Solid State Chem. 190, 24-28.

Artini, C., Pani, M., Lausi, A., Masini, R. \& Costa, G. A. (2014). Inorg. Chem. 53, 10140-10149.

Chavan, S. V. \& Tyagi, A. K. (2005). Mater. Sci. Eng. A. 404, 57?63. Grover, V. \& Tyagi, A. K. (2004). Mater. Res. Bull. $39,859-866$.

Inaba, H. \& Tagawa, H. (1996). Solid State Ionics, 83, 1-6.

Jadhav, L. D., Chourashiya, M. G., Jamale, A. P., Chavan, A. U. \& Patil, S. P. (2010). J. Alloys Compd. 506, 739-744.

Kilner, J. A. \& Brook, R. J. (1982). Solid State Ionics, 6. 237-252.

Kilner, J. A. \& Burriel, M. (2014). Annu. Rev. Mater. Res. 44. 365-393.

Kilner, JA. (1983). Solid State Ionics, 8, 201-207

Kilner, J. A. \& Waters, C. D. (1982). Solid State Ionics, 6, 253-259.

Kim, D. J. (1989). J. Am. Ceram. Soc. 72,1415

Kossoy, A., Wang, Q., Korobko, R., Grover, V., Feldman, Y., Wachtel, E., Tyagi, A. K., Frenkel, A. K. \& Lubomirsky, I. (2013). Phys. Rev. B 87, 054101.

Larson, A. C. \& Von Dreele, R. B. (2004). GSAS. Report LAUR 86-748. Los Alamos National Laboratory, New Mexico, USA.

Maslen, E. N., Streltsov, V. A. \& Ishizawa, N. (1996). Acta. Cryst. B52, 414-422.

Nakagawa, T., Osuki, T., Yamamoto, T. A., Kitauji, Y., Kano, M., Katsura, M. \& Emura, S. (2001). J. Synchrotron Rad. 8, 740-742.

Ou, D. R., Mori, T., Ye, F., Zou, J., Auchterlonie, G. \& Drennan, J. (2007). J. Electrochem. Soc. 154, B616-B622.

Rushton, M. J. D., Chroneos, A., Skinner, S. J., Kilner, J. A. \& Grimes, R. W. (2013). Solid State Ionics, 230, 37-42.

Shannon, R. D. \& Prewitt, C. T. (1968). Acta. Cryst. B25, 925. Steele, B. C. H. (2000). Solid State Ionics, 129, 95-110.

Tianshu, Z., Hing, P., Huang, H. \& Kilner, J. (2002). Solid State Ionics, 148, 567-573.

Tyagi, A. K., Ambekar, B. R. \& Mathews, M. D. (2002). J. Alloys Compd. 337, $277-281$.

Wang, Z., Kale, G. M. \& Ghadiri, M. (2012). Chem. Eng. J. 198-199, 149-153.

Wang, Z., Kale, G. M. \& Ghadiri, M. (2012). J. Am. Ceram. Soc. 95, 3124-3129. 


\section{Figure 1}

Rietveld refinement of $\mathrm{Ce}_{0.8} \mathrm{Ho}_{0.1} \mathrm{Gd}_{0.1} \mathrm{O}_{1.9}$ at $873 \mathrm{~K}$, confirming the presence of single phase fluorite.

\section{Figure 2}

XRD patterns of $\mathrm{Ce}_{0.8} \mathrm{Ho}_{0.1} \mathrm{Gd}_{0.1} \mathrm{O}_{1.9}$ at selected temperatures.

\section{Figure 3}

Lattice parameter of $\mathrm{Ce}_{0.8} \mathrm{Ho}_{0.1} \mathrm{Gd}_{0.1} \mathrm{O}_{1.9}$ as a function of temperature.

\section{Figure 4}

Percentage of lattice thermal expansion of $\mathrm{Ce}_{0.8} \mathrm{Ho}_{0.1} \mathrm{Gd}_{0.1} \mathrm{O}_{1.9}$ as a function of temperature. 


\section{supporting information}

\section{Crystal structure of $\mathrm{Ce}_{0.8} \mathrm{Ho}_{0.1} \mathrm{Gd}_{0.1} \mathrm{O}_{1.9}$ between $295 \mathrm{~K}$ and $1023 \mathrm{~K}$}

\section{Computing details}

Program(s) used to refine structure: GSAS; molecular graphics: Atoms 6.2; software used to prepare material for publication: enCIFer.

\section{(HO500_25_publ)}

\section{Crystal data}

$\mathrm{Ce} 0.8 \mathrm{Ho} 0.1 \mathrm{Gd} 0.1 \mathrm{O}_{1.9}$

$M_{r}=174.71$

Cubic, $F M \overline{3} M$

Hall symbol: $\mathrm{F} \mathrm{m}-3 \mathrm{~m}$

$a=5.4214(7) \AA$

$V=159.34(6) \AA^{3}$

$Z=1$

Data collection

PANalytical X'Pert XRD

diffractometer

$\mathrm{Cu}$ monochromator

Specimen mounting: flat plate holder

\section{Refinement}

Least-squares matrix: full

$R_{\mathrm{p}}=0.041$

$R_{\mathrm{wp}}=0.052$

$R_{\exp }=0.044$

$R\left(F^{2}\right)=0.04859$
$\mathrm{Cu} \mathrm{K} \alpha, \mathrm{Cu} \mathrm{K} \alpha$ radiation, $\lambda=1.5406,1.5418 \AA$

$T=298 \mathrm{~K}$

Particle morphology: powder

pale yellow

Specimen preparation: Prepared at $973 \mathrm{~K}$ and $0 \mathrm{kPa}$, cooled at $1 \mathrm{~K} \mathrm{~min}^{-1}$

Data collection mode: reflection

Scan method: step

$2 \theta_{\min }=9.878^{\circ}, 2 \theta_{\max }=99.803^{\circ}, 2 \theta_{\text {step }}=0.033^{\circ}$

Profile function: CW Profile function number 4 with 14 terms Pseudovoigt profile coefficients as parameterized in P. Thompson, D.E. Cox \& J.B. Hastings (1987). J. Appl. Cryst.,20,79-83. Asymmetry correction of L.W. Finger, D.E. Cox \& A. P. Jephcoat (1994). J. Appl. Cryst.,27,892-900. Microstrain broadening by P.W. Stephens, (1999). J. Appl. Cryst.,32,281-289. \#1(GU) $=1127.450 \# 2(\mathrm{GV})=$ $* * * * * * * * 3(\mathrm{GW})=2992.640 \# 4(\mathrm{GP})=1256.950$ $\# 5(\mathrm{LX})=46.702 \# 6($ ptec $)=3.53 \# 7$ (trns) $=0.00$ $\# 8(\mathrm{shft})=0.0000 \# 9(\mathrm{sfec})=0.00 \# 10(\mathrm{~S} / \mathrm{L})=0.0050$ $\# 11(\mathrm{H} / \mathrm{L})=0.0005 \# 12($ eta $)=0.0000 \# 13(\mathrm{~S} 400)=$ $0.0 \mathrm{E}+00 \# 14(\mathrm{~S} 220)=0.0 \mathrm{E}+00$ Peak tails are ignored where the intensity is below 0.0001 times the peak Aniso. broadening axis 0.00 .01 .0

24 parameters

0 restraints

$(\Delta / \sigma)_{\max }=0.03$

Background function: GSAS Background function number 1 with 20 terms. Shifted Chebyshev function of 1st kind 1: 395.922 2: 128.0913 : -7.03946 4: 6.67325 5: -3.29283 6: 0.252425 7: -3.288958 : 3.40089 9: 3.97722 10: -3.25194 11: -7.20781 12: -4.57777 13: 5.00053 14: 1.86540 15: -8.0264216 : -5.79388 17: 0.967461 18: -2.36138 19: 4.3541420 : $-0.667179$ 
Special details

Refinement. Resolution

Fractional atomic coordinates and isotropic or equivalent isotropic displacement parameters $\left(\AA^{2}\right)$

\begin{tabular}{llllll}
\hline & $x$ & $y$ & $z$ & $U_{\text {iso }} * / U_{\text {eq }}$ & Occ. $(<1)$ \\
\hline CE & 0.0 & 0.0 & 0.0 & $0.00304^{*}$ & 0.8 \\
GD & 0.0 & 0.0 & 0.0 & $0.00304^{*}$ & 0.1 \\
HO & 0.0 & 0.0 & 0.0 & $0.00304^{*}$ & 0.1 \\
O & 0.25 & 0.25 & 0.25 & $0.00051^{*}$ & 0.95 \\
\hline
\end{tabular}

Geometric parameters $\left(\AA,{ }^{\circ}\right)$

\begin{tabular}{|c|c|c|c|}
\hline $\mathrm{CE}-\mathrm{CE}^{\mathrm{i}}$ & 3.8335 (4) & $\mathrm{GD}-\mathrm{HO}^{\mathrm{v}}$ & 3.8335 (4) \\
\hline $\mathrm{CE}-\mathrm{CE}^{\mathrm{ii}}$ & $3.8335(4)$ & $\mathrm{GD}-\mathrm{HO}^{\mathrm{vi}}$ & 3.8335 (4) \\
\hline $\mathrm{CE}-\mathrm{CE} \mathrm{E}^{\mathrm{iii}}$ & 3.8335 (4) & $\mathrm{GD}-\mathrm{HO}^{\mathrm{vii}}$ & 3.8335 (4) \\
\hline $\mathrm{CE}-\mathrm{CE} \mathrm{E}^{\mathrm{iv}}$ & 3.8335 (4) & $\mathrm{GD}-\mathrm{HO}^{\mathrm{viii}}$ & 3.8335 (4) \\
\hline $\mathrm{CE}-\mathrm{CE}^{\mathrm{v}}$ & 3.8335 (4) & $\mathrm{GD}-\mathrm{HO}^{\mathrm{ix}}$ & $3.8335(5)$ \\
\hline $\mathrm{CE}-\mathrm{CE}^{\mathrm{vi}}$ & $3.8335(4)$ & $\mathrm{GD}-\mathrm{HO}^{\mathrm{x}}$ & $3.8335(5)$ \\
\hline $\mathrm{CE}-\mathrm{CE}^{\mathrm{vii}}$ & $3.8335(4)$ & $\mathrm{GD}-\mathrm{HO}^{\mathrm{xi}}$ & $3.8335(5)$ \\
\hline $\mathrm{CE}-\mathrm{CE}^{\mathrm{viii}}$ & 3.8335 (4) & $\mathrm{GD}-\mathrm{HO}^{\mathrm{xii}}$ & $3.8335(5)$ \\
\hline $\mathrm{CE}-\mathrm{CE}^{\mathrm{ix}}$ & $3.8335(5)$ & $\mathrm{GD}-\mathrm{O}$ & $2.3475(2)$ \\
\hline $\mathrm{CE}-\mathrm{CE}^{\mathrm{x}}$ & $3.8335(5)$ & $\mathrm{GD}-\mathrm{O}^{\mathrm{xiii}}$ & $2.3475(2)$ \\
\hline $\mathrm{CE}-\mathrm{CE}^{\mathrm{xi}}$ & $3.8335(5)$ & $\mathrm{GD}-\mathrm{O}^{\mathrm{xiv}}$ & $2.3475(2)$ \\
\hline $\mathrm{CE}-\mathrm{CE}^{\mathrm{xii}}$ & $3.8335(5)$ & $\mathrm{GD}-\mathrm{O}^{\mathrm{xv}}$ & $2.3475(2)$ \\
\hline $\mathrm{CE}-\mathrm{GD}^{\mathrm{i}}$ & 3.8335 (4) & $\mathrm{GD}-\mathrm{O}^{\mathrm{xvi}}$ & $2.3475(2)$ \\
\hline $\mathrm{CE}-\mathrm{GD}^{\mathrm{ii}}$ & 3.8335 (4) & $\mathrm{GD}-\mathrm{O}^{\mathrm{xvii}}$ & $2.3475(2)$ \\
\hline $\mathrm{CE}-\mathrm{GD}^{\mathrm{iii}}$ & 3.8335 (4) & $\mathrm{GD}-\mathrm{O}^{\mathrm{xviii}}$ & $2.3475(2)$ \\
\hline $\mathrm{CE}-\mathrm{GD}^{\mathrm{iv}}$ & 3.8335 (4) & $\mathrm{GD}-\mathrm{O}^{\mathrm{xix}}$ & $2.3475(2)$ \\
\hline $\mathrm{CE}-\mathrm{GD}^{\mathrm{v}}$ & 3.8335 (4) & $\mathrm{HO}-\mathrm{CE}^{\mathrm{i}}$ & 3.8335 (4) \\
\hline $\mathrm{CE}-\mathrm{GD}^{\mathrm{vi}}$ & 3.8335 (4) & $\mathrm{HO}-\mathrm{CE}^{\mathrm{ii}}$ & $3.8335(4)$ \\
\hline $\mathrm{CE}-\mathrm{GD}^{\mathrm{vii}}$ & $3.8335(4)$ & $\mathrm{HO}-\mathrm{CE}^{\mathrm{iii}}$ & 3.8335 (4) \\
\hline $\mathrm{CE}-\mathrm{GD}^{\text {viii }}$ & 3.8335 (4) & $\mathrm{HO}-\mathrm{CE}^{\mathrm{iv}}$ & 3.8335 (4) \\
\hline $\mathrm{CE}-\mathrm{GD}^{\mathrm{ix}}$ & $3.8335(5)$ & $\mathrm{HO}-\mathrm{CE}^{\mathrm{v}}$ & 3.8335 (4) \\
\hline $\mathrm{CE}-\mathrm{GD}^{\mathrm{x}}$ & $3.8335(5)$ & $\mathrm{HO}-\mathrm{CE}^{\mathrm{vi}}$ & 3.8335 (4) \\
\hline $\mathrm{CE}-\mathrm{GD}^{\mathrm{xi}}$ & $3.8335(5)$ & $\mathrm{HO}-\mathrm{CE}^{\mathrm{vii}}$ & 3.8335 (4) \\
\hline $\mathrm{CE}-\mathrm{GD}^{\mathrm{xii}}$ & $3.8335(5)$ & $\mathrm{HO}-\mathrm{CE}^{\mathrm{viii}}$ & 3.8335 (4) \\
\hline $\mathrm{CE}-\mathrm{HO}^{\mathrm{i}}$ & 3.8335 (4) & $\mathrm{HO}-\mathrm{CE}^{\mathrm{ix}}$ & $3.8335(5)$ \\
\hline $\mathrm{CE}-\mathrm{HO}^{\mathrm{ii}}$ & 3.8335 (4) & $\mathrm{HO}-\mathrm{CE}^{\mathrm{x}}$ & $3.8335(5)$ \\
\hline $\mathrm{CE}-\mathrm{HO}^{\mathrm{iii}}$ & 3.8335 (4) & $\mathrm{HO}-\mathrm{CE}^{\mathrm{xi}}$ & $3.8335(5)$ \\
\hline $\mathrm{CE}-\mathrm{HO}^{\mathrm{iv}}$ & 3.8335 (4) & $\mathrm{HO}-\mathrm{CE}^{\mathrm{xii}}$ & $3.8335(5)$ \\
\hline $\mathrm{CE}-\mathrm{HO}^{\mathrm{v}}$ & 3.8335 (4) & $\mathrm{HO}-\mathrm{GD}^{\mathrm{i}}$ & 3.8335 (4) \\
\hline $\mathrm{CE}-\mathrm{HO}^{\mathrm{vi}}$ & $3.8335(4)$ & $\mathrm{HO}-\mathrm{GD}^{\mathrm{ii}}$ & 3.8335 (4) \\
\hline $\mathrm{CE}-\mathrm{HO}^{\mathrm{vii}}$ & 3.8335 (4) & $\mathrm{HO}-\mathrm{GD}^{\mathrm{iii}}$ & 3.8335 (4) \\
\hline $\mathrm{CE}-\mathrm{HO}^{\text {viii }}$ & 3.8335 (4) & $\mathrm{HO}-\mathrm{GD}^{\mathrm{iv}}$ & 3.8335 (4) \\
\hline $\mathrm{CE}-\mathrm{HO}^{\mathrm{ix}}$ & $3.8335(5)$ & $\mathrm{HO}-\mathrm{GD}^{\mathrm{v}}$ & 3.8335 (4) \\
\hline $\mathrm{CE}-\mathrm{HO}^{\mathrm{x}}$ & $3.8335(5)$ & $\mathrm{HO}-\mathrm{GD}^{\mathrm{vi}}$ & 3.8335 (4) \\
\hline $\mathrm{CE}-\mathrm{HO}^{\mathrm{xi}}$ & $3.8335(5)$ & $\mathrm{HO}-\mathrm{GD}^{\mathrm{vii}}$ & 3.8335 (4) \\
\hline $\mathrm{CE}-\mathrm{HO}^{\mathrm{xii}}$ & $3.8335(5)$ & $\mathrm{HO}-\mathrm{GD}^{\mathrm{viii}}$ & 3.8335 (4) \\
\hline $\mathrm{CE}-\mathrm{O}$ & $2.3475(2)$ & $\mathrm{HO}-\mathrm{GD}^{\mathrm{ix}}$ & 3.8335 (5) \\
\hline $\mathrm{CE}-\mathrm{O}^{\mathrm{xiii}}$ & $2.3475(2)$ & $\mathrm{HO}-\mathrm{GD}^{\mathrm{x}}$ & $3.8335(5)$ \\
\hline
\end{tabular}




\begin{tabular}{|c|c|c|c|}
\hline $\mathrm{CE}-\mathrm{O}^{\mathrm{xiv}}$ & $2.3475(2)$ & $\mathrm{HO}-\mathrm{GD}^{\mathrm{xi}}$ & $3.8335(5)$ \\
\hline $\mathrm{CE}-\mathrm{O}^{\mathrm{xv}}$ & $2.3475(2)$ & $\mathrm{HO}-\mathrm{GD}^{\mathrm{xii}}$ & $3.8335(5)$ \\
\hline $\mathrm{CE}-\mathrm{O}^{\mathrm{xvi}}$ & $2.3475(2)$ & $\mathrm{HO}-\mathrm{HO}^{\mathrm{i}}$ & $3.8335(4)$ \\
\hline $\mathrm{CE}-\mathrm{O}^{\mathrm{xvii}}$ & $2.3475(2)$ & $\mathrm{HO}-\mathrm{HO}^{\mathrm{ii}}$ & $3.8335(4)$ \\
\hline $\mathrm{CE}-\mathrm{O}^{\mathrm{xviii}}$ & $2.3475(2)$ & $\mathrm{HO}-\mathrm{HO}^{\mathrm{iii}}$ & $3.8335(4)$ \\
\hline $\mathrm{CE}-\mathrm{O}^{\mathrm{xix}}$ & $2.3475(2)$ & $\mathrm{HO}-\mathrm{HO}^{\mathrm{iv}}$ & $3.8335(4)$ \\
\hline $\mathrm{GD}-\mathrm{CE}^{\mathrm{i}}$ & $3.8335(4)$ & $\mathrm{HO}-\mathrm{HO}^{v}$ & $3.8335(4)$ \\
\hline $\mathrm{GD}-\mathrm{CE}^{\mathrm{ii}}$ & $3.8335(4)$ & $\mathrm{HO}-\mathrm{HO}^{\mathrm{vi}}$ & $3.8335(4)$ \\
\hline $\mathrm{GD}-\mathrm{CE}^{\mathrm{iii}}$ & $3.8335(4)$ & $\mathrm{HO}-\mathrm{HO}^{\mathrm{vii}}$ & $3.8335(4)$ \\
\hline $\mathrm{GD}-\mathrm{CE}^{\mathrm{iv}}$ & $3.8335(4)$ & $\mathrm{HO}-\mathrm{HO}^{\text {viii }}$ & $3.8335(4)$ \\
\hline $\mathrm{GD}-\mathrm{CE}^{\mathrm{v}}$ & $3.8335(4)$ & $\mathrm{HO}-\mathrm{HO}^{\mathrm{ix}}$ & $3.8335(5)$ \\
\hline $\mathrm{GD}-\mathrm{CE}^{\mathrm{vi}}$ & $3.8335(4)$ & $\mathrm{HO}-\mathrm{HO}^{\mathrm{x}}$ & $3.8335(5)$ \\
\hline $\mathrm{GD}-\mathrm{CE}^{\mathrm{vii}}$ & $3.8335(4)$ & $\mathrm{HO}-\mathrm{HO}^{\mathrm{xi}}$ & $3.8335(5)$ \\
\hline $\mathrm{GD}-\mathrm{CE}^{\mathrm{viii}}$ & $3.8335(4)$ & $\mathrm{HO}-\mathrm{HO}^{\mathrm{xii}}$ & $3.8335(5)$ \\
\hline $\mathrm{GD}-\mathrm{CE}^{\mathrm{ix}}$ & $3.8335(5)$ & $\mathrm{HO}-\mathrm{O}$ & $2.3475(2)$ \\
\hline $\mathrm{GD}-\mathrm{CE}^{\mathrm{x}}$ & $3.8335(5)$ & $\mathrm{HO}-\mathrm{O}^{\mathrm{xiii}}$ & $2.3475(2)$ \\
\hline $\mathrm{GD}-\mathrm{CE}^{\mathrm{xi}}$ & $3.8335(5)$ & $\mathrm{HO}-\mathrm{O}^{\mathrm{xiv}}$ & $2.3475(2)$ \\
\hline $\mathrm{GD}-\mathrm{CE}^{\mathrm{xii}}$ & $3.8335(5)$ & $\mathrm{HO}-\mathrm{O}^{\mathrm{xv}}$ & $2.3475(2)$ \\
\hline $\mathrm{GD}-\mathrm{GD}^{\mathrm{i}}$ & $3.8335(4)$ & $\mathrm{HO}-\mathrm{O}^{\mathrm{xvi}}$ & $2.3475(2)$ \\
\hline $\mathrm{GD}-\mathrm{GD}^{\mathrm{ii}}$ & $3.8335(4)$ & $\mathrm{HO}-\mathrm{O}^{\mathrm{xvii}}$ & $2.3475(2)$ \\
\hline $\mathrm{GD}-\mathrm{GD}^{\mathrm{iii}}$ & $3.8335(4)$ & $\mathrm{HO}-\mathrm{O}^{\mathrm{xviii}}$ & $2.3475(2)$ \\
\hline $\mathrm{GD}-\mathrm{GD}^{\mathrm{iv}}$ & $3.8335(4)$ & $\mathrm{HO}-\mathrm{O}^{\mathrm{xix}}$ & $2.3475(2)$ \\
\hline $\mathrm{GD}-\mathrm{GD}^{\mathrm{v}}$ & $3.8335(4)$ & $\mathrm{O}-\mathrm{CE}$ & $2.3475(2)$ \\
\hline $\mathrm{GD}-\mathrm{GD}^{\mathrm{vi}}$ & $3.8335(4)$ & $\mathrm{O}-\mathrm{CE}^{\mathrm{iv}}$ & $2.3475(2)$ \\
\hline GD-GD ${ }^{\text {vii }}$ & $3.8335(4)$ & $\mathrm{O}-\mathrm{CE}^{\mathrm{viii}}$ & $2.3475(2)$ \\
\hline GD-GD ${ }^{\text {viii }}$ & $3.8335(4)$ & $\mathrm{O}-\mathrm{CE}^{\mathrm{xii}}$ & $2.3475(2)$ \\
\hline $\mathrm{GD}-\mathrm{GD}^{\mathrm{ix}}$ & $3.8335(5)$ & $\mathrm{O}-\mathrm{GD}$ & $2.3475(2)$ \\
\hline $\mathrm{GD}-\mathrm{GD}^{\mathrm{x}}$ & $3.8335(5)$ & $\mathrm{O}-\mathrm{GD}^{\mathrm{iv}}$ & $2.3475(2)$ \\
\hline $\mathrm{GD}-\mathrm{GD}^{\mathrm{xi}}$ & $3.8335(5)$ & $\mathrm{O}-\mathrm{GD}^{\mathrm{viii}}$ & $2.3475(2)$ \\
\hline $\mathrm{GD}-\mathrm{GD}^{\mathrm{xii}}$ & $3.8335(5)$ & $\mathrm{O}-\mathrm{GD}^{\mathrm{xii}}$ & $2.3475(2)$ \\
\hline $\mathrm{GD}-\mathrm{HO}^{\mathrm{i}}$ & $3.8335(4)$ & $\mathrm{O}-\mathrm{HO}$ & $2.3475(2)$ \\
\hline $\mathrm{GD}-\mathrm{HO}^{\mathrm{ii}}$ & $3.8335(4)$ & $\mathrm{O}-\mathrm{HO}^{\mathrm{iv}}$ & $2.3475(2)$ \\
\hline $\mathrm{GD}-\mathrm{HO}^{\mathrm{iii}}$ & $3.8335(4)$ & $\mathrm{O}-\mathrm{HO}^{\text {viii }}$ & $2.3475(2)$ \\
\hline $\mathrm{GD}-\mathrm{HO}^{\mathrm{iv}}$ & $3.8335(4)$ & $\mathrm{O}-\mathrm{HO}^{\mathrm{xii}}$ & $2.3475(2)$ \\
\hline $\mathrm{O}-\mathrm{CE}-\mathrm{O}^{\mathrm{xiii}}$ & $70.529(10)$ & $\mathrm{O}^{\mathrm{xv}}-\mathrm{HO}-\mathrm{O}^{\mathrm{xvii}}$ & $70.529(5)$ \\
\hline $\mathrm{O}-\mathrm{CE}-\mathrm{O}^{\mathrm{xiv}}$ & $70.529(5)$ & $\mathrm{O}^{\mathrm{xv}}-\mathrm{HO}-\mathrm{O}^{\mathrm{xviii}}$ & $70.529(10)$ \\
\hline $\mathrm{O}-\mathrm{CE}-\mathrm{O}^{\mathrm{xv}}$ & $70.529(5)$ & $\mathrm{O}^{\mathrm{xv}}-\mathrm{HO}-\mathrm{O}^{\mathrm{xix}}$ & $109.471(5)$ \\
\hline $\mathrm{O}-\mathrm{CE}-\mathrm{O}^{\mathrm{xvi}}$ & $109.471(5)$ & $\mathrm{O}^{\mathrm{xvi}}-\mathrm{HO}-\mathrm{O}^{\mathrm{xvii}}$ & $109.471(5)$ \\
\hline $\mathrm{O}-\mathrm{CE}-\mathrm{O}^{\mathrm{xvii}}$ & $109.471(10)$ & $\mathrm{O}^{\mathrm{xvi}}-\mathrm{HO}-\mathrm{O}^{\mathrm{xviii}}$ & $109.471(10)$ \\
\hline $\mathrm{O}-\mathrm{CE}-\mathrm{O}^{\mathrm{xviii}}$ & $109.471(5)$ & $\mathrm{O}^{\mathrm{xvi}}-\mathrm{HO}-\mathrm{O}^{\mathrm{xix}}$ & $70.529(5)$ \\
\hline $\mathrm{O}-\mathrm{CE}-\mathrm{O}^{\mathrm{xix}}$ & 180.0 & $\mathrm{O}^{\mathrm{xvii}}-\mathrm{HO}-\mathrm{O}^{\mathrm{xviii}}$ & $109.471(5)$ \\
\hline $\mathrm{O}^{\mathrm{xiii}}-\mathrm{CE}-\mathrm{O}^{\mathrm{xiv}}$ & $109.471(5)$ & $\mathrm{O}^{\mathrm{xvii}}-\mathrm{HO}-\mathrm{O}^{\mathrm{xix}}$ & $70.529(10)$ \\
\hline $\mathrm{O}^{\mathrm{xiii}}-\mathrm{CE}-\mathrm{O}^{\mathrm{xv}}$ & $109.471(5)$ & $\mathrm{O}^{\mathrm{xviii}}-\mathrm{HO}-\mathrm{O}^{\mathrm{xix}}$ & $70.529(5)$ \\
\hline $\mathrm{O}^{\mathrm{xiii}}-\mathrm{CE}-\mathrm{O}^{\mathrm{xvi}}$ & $70.529(5)$ & $\mathrm{CE}-\mathrm{O}-\mathrm{CE}^{\mathrm{iv}}$ & $109.471(5)$ \\
\hline $\mathrm{O}^{\mathrm{xiii}}-\mathrm{CE}-\mathrm{O}^{\mathrm{xvii}}$ & 180.0 & $\mathrm{CE}-\mathrm{O}-\mathrm{CE}^{\mathrm{viii}}$ & $109.471(5)$ \\
\hline $\mathrm{O}^{\mathrm{xiii}}-\mathrm{CE}-\mathrm{O}^{\mathrm{xviii}}$ & $70.529(5)$ & $\mathrm{CE}-\mathrm{O}-\mathrm{CE}^{\mathrm{xii}}$ & $109.471(10)$ \\
\hline $\mathrm{O}^{\mathrm{xiii}}-\mathrm{CE}-\mathrm{O}^{\mathrm{xix}}$ & $109.471(10)$ & $\mathrm{CE}-\mathrm{O}-\mathrm{GD}$ & 0.0 \\
\hline $\mathrm{O}^{\mathrm{xiv}}-\mathrm{CE}-\mathrm{O}^{\mathrm{xv}}$ & $109.471(10)$ & $\mathrm{CE}-\mathrm{O}-\mathrm{GD}^{\mathrm{iv}}$ & $109.471(5)$ \\
\hline $\mathrm{O}^{\mathrm{xiv}}-\mathrm{CE}-\mathrm{O}^{\mathrm{xvi}}$ & $70.529(10)$ & $\mathrm{CE}-\mathrm{O}-\mathrm{GD}^{\mathrm{viii}}$ & $109.471(5)$ \\
\hline $\mathrm{O}^{\mathrm{xiv}}-\mathrm{CE}-\mathrm{O}^{\mathrm{xvii}}$ & $70.529(5)$ & $\mathrm{CE}-\mathrm{O}-\mathrm{GD}^{\mathrm{xii}}$ & $109.471(10)$ \\
\hline $\mathrm{O}^{\mathrm{xiv}}-\mathrm{CE}-\mathrm{O}^{\mathrm{xviii}}$ & 180.0 & $\mathrm{CE}-\mathrm{O}-\mathrm{HO}$ & 0.0 \\
\hline
\end{tabular}




\begin{tabular}{|c|c|c|c|}
\hline $\mathrm{O}^{\mathrm{xiv}}-\mathrm{CE}-\mathrm{O}^{\mathrm{xix}}$ & $109.471(5)$ & $\mathrm{CE}-\mathrm{O}-\mathrm{HO}^{\mathrm{iv}}$ & $109.471(5)$ \\
\hline $\mathrm{O}^{\mathrm{xv}}-\mathrm{CE}-\mathrm{O}^{\mathrm{xvi}}$ & 180.0 & $\mathrm{CE}-\mathrm{O}-\mathrm{HO}^{\text {viii }}$ & $109.471(5)$ \\
\hline $\mathrm{O}^{\mathrm{xv}}-\mathrm{CE}-\mathrm{O}^{\mathrm{xvii}}$ & $70.529(5)$ & $\mathrm{CE}-\mathrm{O}-\mathrm{HO}^{\mathrm{xii}}$ & $109.471(10)$ \\
\hline $\mathrm{O}^{\mathrm{xv}}-\mathrm{CE}-\mathrm{O}^{\mathrm{xviii}}$ & $70.529(10)$ & $\mathrm{CE}^{\mathrm{iv}}-\mathrm{O}-\mathrm{CE}^{\mathrm{viii}}$ & $109.471(10)$ \\
\hline $\mathrm{O}^{\mathrm{xv}}-\mathrm{CE}-\mathrm{O}^{\mathrm{xix}}$ & $109.471(5)$ & $C E^{i v}-\mathrm{O}-\mathrm{CE}^{\mathrm{xii}}$ & $109.471(5)$ \\
\hline $\mathrm{O}^{\mathrm{xvi}}-\mathrm{CE}-\mathrm{O}^{\mathrm{xvii}}$ & $109.471(5)$ & $\mathrm{CE}^{\mathrm{iv}}-\mathrm{O}-\mathrm{GD}$ & $109.471(5)$ \\
\hline $\mathrm{O}^{\mathrm{xvi}}-\mathrm{CE}-\mathrm{O}^{\mathrm{xviii}}$ & $109.471(10)$ & $\mathrm{CE}^{\mathrm{iv}}-\mathrm{O}-\mathrm{GD}^{\mathrm{iv}}$ & 0.0 \\
\hline $\mathrm{O}^{\mathrm{xvi}}-\mathrm{CE}-\mathrm{O}^{\mathrm{xix}}$ & $70.529(5)$ & $\mathrm{CE}^{\mathrm{iv}}-\mathrm{O}-\mathrm{GD}^{\text {viii }}$ & $109.471(10)$ \\
\hline $\mathrm{O}^{\mathrm{xvii}}-\mathrm{CE}-\mathrm{O}^{\mathrm{xviii}}$ & $109.471(5)$ & $\mathrm{CE}^{\mathrm{iv}}-\mathrm{O}-\mathrm{GD}^{\mathrm{xii}}$ & $109.471(5)$ \\
\hline $\mathrm{O}^{\mathrm{xvii}}-\mathrm{CE}-\mathrm{O}^{\mathrm{xix}}$ & $70.529(10)$ & $\mathrm{CE}^{\mathrm{iv}}-\mathrm{O}-\mathrm{HO}$ & $109.471(5)$ \\
\hline $\mathrm{O}^{\mathrm{x} \text { viii }}-\mathrm{CE}-\mathrm{O}^{\mathrm{xix}}$ & $70.529(5)$ & $\mathrm{CE}^{\mathrm{iv}}-\mathrm{O}-\mathrm{HO}^{\mathrm{iv}}$ & 0.0 \\
\hline $\mathrm{O}-\mathrm{GD}-\mathrm{O}^{\mathrm{xiii}}$ & $70.529(10)$ & $\mathrm{CE}^{\mathrm{iv}}-\mathrm{O}-\mathrm{HO}^{\text {viii }}$ & $109.471(10)$ \\
\hline $\mathrm{O}-\mathrm{GD}-\mathrm{O}^{\mathrm{xiv}}$ & $70.529(5)$ & $\mathrm{CE}^{\mathrm{iv}}-\mathrm{O}-\mathrm{HO}^{\mathrm{xii}}$ & $109.471(5)$ \\
\hline $\mathrm{O}-\mathrm{GD}-\mathrm{O}^{\mathrm{xv}}$ & $70.529(5)$ & $\mathrm{CE}^{\mathrm{viii}}-\mathrm{O}-\mathrm{CE}^{\mathrm{xii}}$ & $109.471(5)$ \\
\hline $\mathrm{O}-\mathrm{GD}-\mathrm{O}^{\mathrm{xvi}}$ & $109.471(5)$ & 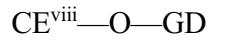 & $109.471(5)$ \\
\hline $\mathrm{O}-\mathrm{GD}-\mathrm{O}^{\mathrm{xvii}}$ & $109.471(10)$ & $\mathrm{CE}^{\mathrm{viii}}-\mathrm{O}-\mathrm{GD}^{\mathrm{iv}}$ & $109.471(10)$ \\
\hline $\mathrm{O}-\mathrm{GD}-\mathrm{O}^{\mathrm{xviii}}$ & $109.471(5)$ & $\mathrm{CE}^{\mathrm{viii}}-\mathrm{O}-\mathrm{GD}^{\mathrm{viii}}$ & 0.0 \\
\hline $\mathrm{O}-\mathrm{GD}-\mathrm{O}^{\mathrm{xix}}$ & 180.0 & $\mathrm{CE}^{\mathrm{viii}}-\mathrm{O}-\mathrm{GD}^{\mathrm{xii}}$ & $109.471(5)$ \\
\hline $\mathrm{O}^{\mathrm{xii}}-\mathrm{GD}-\mathrm{O}^{\mathrm{xiv}}$ & $109.471(5)$ & $\mathrm{CE}^{\mathrm{viii}}-\mathrm{O}-\mathrm{HO}$ & $109.471(5)$ \\
\hline $\mathrm{O}^{\mathrm{xiii}}-\mathrm{GD}-\mathrm{O}^{\mathrm{xv}}$ & $109.471(5)$ & $\mathrm{CE}^{\text {viii }}-\mathrm{O}-\mathrm{HO}^{\mathrm{iv}}$ & $109.471(10)$ \\
\hline $\mathrm{O}^{\mathrm{xiii}}-\mathrm{GD}-\mathrm{O}^{\mathrm{xvi}}$ & $70.529(5)$ & $\mathrm{CE}^{\mathrm{viii}}-\mathrm{O}-\mathrm{HO}^{\text {viii }}$ & 0.0 \\
\hline $\mathrm{O}^{\mathrm{xiii}}-\mathrm{GD}-\mathrm{O}^{\mathrm{xvii}}$ & 180.0 & $\mathrm{CE}^{\mathrm{viii}}-\mathrm{O}-\mathrm{HO}^{\mathrm{xii}}$ & $109.471(5)$ \\
\hline $\mathrm{O}^{\mathrm{xiii}}-\mathrm{GD}-\mathrm{O}^{\mathrm{xviii}}$ & $70.529(5)$ & $\mathrm{CE}^{\mathrm{xii}}-\mathrm{O}-\mathrm{GD}$ & $109.471(10)$ \\
\hline $\mathrm{O}^{\mathrm{xiii}}-\mathrm{GD}-\mathrm{O}^{\mathrm{xix}}$ & $109.471(10)$ & $\mathrm{CE}^{\mathrm{xii}}-\mathrm{O}-\mathrm{GD}^{\mathrm{iv}}$ & $109.471(5)$ \\
\hline $\mathrm{O}^{\mathrm{xiv}}-\mathrm{GD}-\mathrm{O}^{\mathrm{xv}}$ & $109.471(10)$ & $\mathrm{CE}^{\mathrm{xii}}-\mathrm{O}-\mathrm{GD}^{\text {viii }}$ & $109.471(5)$ \\
\hline $\mathrm{O}^{\mathrm{xiv}}-\mathrm{GD}-\mathrm{O}^{\mathrm{xvi}}$ & $70.529(10)$ & $\mathrm{CE}^{\mathrm{xii}}-\mathrm{O}-\mathrm{GD}^{\mathrm{xii}}$ & 0.0 \\
\hline $\mathrm{O}^{\mathrm{xiv}}-\mathrm{GD}-\mathrm{O}^{\mathrm{xvii}}$ & $70.529(5)$ & $\mathrm{CE}^{\mathrm{xii}}-\mathrm{O}-\mathrm{HO}$ & $109.471(10)$ \\
\hline $\mathrm{O}^{\mathrm{xiv}}-\mathrm{GD}-\mathrm{O}^{\mathrm{xviii}}$ & 180.0 & $\mathrm{CE}^{\mathrm{xii}}-\mathrm{O}-\mathrm{HO}^{\mathrm{iv}}$ & $109.471(5)$ \\
\hline $\mathrm{O}^{\mathrm{xiv}}-\mathrm{GD}-\mathrm{O}^{\mathrm{xix}}$ & $109.471(5)$ & $\mathrm{CE}^{\mathrm{xii}}-\mathrm{O}-\mathrm{HO}^{\mathrm{viii}}$ & $109.471(5)$ \\
\hline $\mathrm{O}^{\mathrm{xv}}-\mathrm{GD}-\mathrm{O}^{\mathrm{xvi}}$ & 180.0 & $\mathrm{CE}^{\mathrm{xii}}-\mathrm{O}-\mathrm{HO}^{\mathrm{xii}}$ & 0.0 \\
\hline $\mathrm{O}^{\mathrm{xv}}-\mathrm{GD}-\mathrm{O}^{\mathrm{xvii}}$ & $70.529(5)$ & $\mathrm{GD}-\mathrm{O}-\mathrm{GD}^{\mathrm{iv}}$ & $109.471(5)$ \\
\hline $\mathrm{O}^{\mathrm{xv}}-\mathrm{GD}-\mathrm{O}^{\mathrm{xviii}}$ & $70.529(10)$ & $\mathrm{GD}-\mathrm{O}-\mathrm{GD}^{\text {viii }}$ & $109.471(5)$ \\
\hline $\mathrm{O}^{\mathrm{xv}}-\mathrm{GD}-\mathrm{O}^{\mathrm{xix}}$ & $109.471(5)$ & $\mathrm{GD}-\mathrm{O}-\mathrm{GD}^{\mathrm{xii}}$ & $109.471(10)$ \\
\hline $\mathrm{O}^{\mathrm{xvi}}-\mathrm{GD}-\mathrm{O}^{\mathrm{xvii}}$ & $109.471(5)$ & $\mathrm{GD}-\mathrm{O}-\mathrm{HO}$ & 0.0 \\
\hline $\mathrm{O}^{\mathrm{xvi}}-\mathrm{GD}-\mathrm{O}^{\mathrm{xviii}}$ & $109.471(10)$ & $\mathrm{GD}-\mathrm{O}-\mathrm{HO}^{\mathrm{iv}}$ & $109.471(5)$ \\
\hline $\mathrm{O}^{\mathrm{xvi}}-\mathrm{GD}-\mathrm{O}^{\mathrm{xix}}$ & $70.529(5)$ & $\mathrm{GD}-\mathrm{O}-\mathrm{HO}^{\text {viii }}$ & $109.471(5)$ \\
\hline $\mathrm{O}^{\mathrm{xvii}}-\mathrm{GD}-\mathrm{O}^{\mathrm{xviii}}$ & $109.471(5)$ & $\mathrm{GD}-\mathrm{O}-\mathrm{HO}^{\mathrm{xii}}$ & $109.471(10)$ \\
\hline $\mathrm{O}^{\mathrm{xvii}}-\mathrm{GD}-\mathrm{O}^{\mathrm{xix}}$ & $70.529(10)$ & $\mathrm{GD}^{\mathrm{iv}}-\mathrm{O}-\mathrm{GD}^{\mathrm{viii}}$ & $109.471(10)$ \\
\hline $\mathrm{O}^{\mathrm{xviii}}-\mathrm{GD}-\mathrm{O}^{\mathrm{xix}}$ & $70.529(5)$ & $\mathrm{GD}^{\mathrm{iv}}-\mathrm{O}-\mathrm{GD}^{\mathrm{xii}}$ & $109.471(5)$ \\
\hline $\mathrm{O}-\mathrm{HO}-\mathrm{O}^{\mathrm{xiii}}$ & $70.529(10)$ & $\mathrm{GD}^{\mathrm{iv}}-\mathrm{O}-\mathrm{HO}$ & $109.471(5)$ \\
\hline $\mathrm{O}-\mathrm{HO}-\mathrm{O}^{\mathrm{xiv}}$ & $70.529(5)$ & $\mathrm{GD}^{\mathrm{iv}}-\mathrm{O}-\mathrm{HO}^{\mathrm{iv}}$ & 0.0 \\
\hline $\mathrm{O}-\mathrm{HO}-\mathrm{O}^{\mathrm{xv}}$ & $70.529(5)$ & $\mathrm{GD}^{\mathrm{iv}}-\mathrm{O}-\mathrm{HO}^{\text {viii }}$ & $109.471(10)$ \\
\hline $\mathrm{O}-\mathrm{HO}-\mathrm{O}^{\mathrm{xvi}}$ & $109.471(5)$ & $\mathrm{GD}^{\mathrm{iv}}-\mathrm{O}-\mathrm{HO}^{\mathrm{xii}}$ & $109.471(5)$ \\
\hline $\mathrm{O}-\mathrm{HO}-\mathrm{O}^{\mathrm{xvii}}$ & $109.471(10)$ & $\mathrm{GD}^{\mathrm{viii}}-\mathrm{O}-\mathrm{GD}^{\mathrm{xii}}$ & $109.471(5)$ \\
\hline $\mathrm{O}-\mathrm{HO}-\mathrm{O}^{\mathrm{xviii}}$ & $109.471(5)$ & 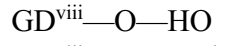 & $109.471(5)$ \\
\hline $\mathrm{O}-\mathrm{HO}-\mathrm{O}^{\mathrm{xix}}$ & 180.0 & $\mathrm{GD}^{\mathrm{vii}}-\mathrm{O}-\mathrm{HO}^{\mathrm{iv}}$ & $109.471(10)$ \\
\hline $\mathrm{O}^{\mathrm{xiii}}-\mathrm{HO}-\mathrm{O}^{\mathrm{xiv}}$ & $109.471(5)$ & $\mathrm{GD}^{\text {viii }}-\mathrm{O}-\mathrm{HO}^{\text {viii }}$ & 0.0 \\
\hline $\mathrm{O}^{\mathrm{xiii}}-\mathrm{HO}-\mathrm{O}^{\mathrm{xv}}$ & $109.471(5)$ & $\mathrm{GD}^{\mathrm{viii}}-\mathrm{O}-\mathrm{HO}^{\mathrm{xii}}$ & $109.471(5)$ \\
\hline $\mathrm{O}^{\mathrm{xii}}-\mathrm{HO}-\mathrm{O}^{\mathrm{xvi}}$ & $70.529(5)$ & $\mathrm{GD}^{\mathrm{xii}}-\mathrm{O}-\mathrm{HO}$ & $109.471(10)$ \\
\hline $\mathrm{O}^{\mathrm{xiii}}-\mathrm{HO}-\mathrm{O}^{\mathrm{xvii}}$ & 180.0 & $\mathrm{GD}^{\mathrm{xii}}-\mathrm{O}-\mathrm{HO}^{\mathrm{iv}}$ & $109.471(5)$ \\
\hline $\mathrm{O}^{\mathrm{xiii}}-\mathrm{HO}-\mathrm{O}^{\mathrm{xviii}}$ & $70.529(5)$ & $\mathrm{GD}^{\mathrm{xii}}-\mathrm{O}-\mathrm{HO}^{\mathrm{vii}}$ & $109.471(5)$ \\
\hline $\mathrm{O}^{\mathrm{xiii}}-\mathrm{HO}-\mathrm{O}^{\mathrm{xix}}$ & $109.471(10)$ & $\mathrm{GD}^{\mathrm{xii}}-\mathrm{O}-\mathrm{HO}^{\mathrm{xii}}$ & 0.0 \\
\hline
\end{tabular}


supporting information

\begin{tabular}{|c|c|c|c|}
\hline $\mathrm{O}^{\mathrm{xiv}}-\mathrm{HO}-\mathrm{O}^{\mathrm{xv}}$ & $109.471(10)$ & $\mathrm{HO}-\mathrm{O}-\mathrm{HO}^{\mathrm{iv}}$ & $109.471(5)$ \\
\hline $\mathrm{O}^{\mathrm{xiv}}-\mathrm{HO}-\mathrm{O}^{\mathrm{xvi}}$ & $70.529(10)$ & $\mathrm{HO}-\mathrm{O}-\mathrm{HO}^{\text {viii }}$ & $109.471(5)$ \\
\hline $\mathrm{O}^{\mathrm{xiv}}-\mathrm{HO}-\mathrm{O}^{\mathrm{xvii}}$ & $70.529(5)$ & $\mathrm{HO}-\mathrm{O}-\mathrm{HO}^{\mathrm{xii}}$ & $109.471(10)$ \\
\hline $\mathrm{O}^{\mathrm{xiv}}-\mathrm{HO}-\mathrm{O}^{\mathrm{xviii}}$ & 180.0 & $\mathrm{HO}^{\mathrm{iv}}-\mathrm{O}-\mathrm{HO}^{\text {viii }}$ & $109.471(10)$ \\
\hline $\mathrm{O}^{\mathrm{xiv}}-\mathrm{HO}-\mathrm{O}^{\mathrm{xix}}$ & $109.471(5)$ & $\mathrm{HO}^{\mathrm{iv}}-\mathrm{O}-\mathrm{HO}^{\mathrm{xii}}$ & $109.471(5)$ \\
\hline $\mathrm{O}^{\mathrm{xv}}-\mathrm{HO}-\mathrm{O}^{\mathrm{xvi}}$ & 180.0 & 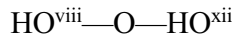 & $109.471(5)$ \\
\hline
\end{tabular}

Symmetry codes: (i) $x, y-1 / 2, z-1 / 2$; (ii) $x, y-1 / 2, z+1 / 2$; (iii) $x, y+1 / 2, z-1 / 2$; (iv) $x, y+1 / 2, z+1 / 2$; (v) $x-1 / 2, y, z-1 / 2$; (vi) $x-1 / 2, y, z+1 / 2$; (vii) $x+1 / 2, y$, $z-1 / 2$; (viii) $x+1 / 2, y, z+1 / 2$; (ix) $x-1 / 2, y-1 / 2, z$; (x) $x-1 / 2, y+1 / 2, z$; (xi) $x+1 / 2, y-1 / 2, z$; (xii) $x+1 / 2, y+1 / 2, z$; (xiii) $x, y,-z$; (xiv) $-z, x, y$; (xv) $y,-z, x$; (xvi) $-z, x,-y$; (xvii) $-y,-z, x$; (xviii) $y,-z,-x$; (xix) $-x,-y,-z$. 